\title{
Multiple parasitic fibroids in different locations- a case report
}

\author{
Rajkumaralal R.D, Yu S.L \\ Department of Obstetrics and Gynaecology, Singapore General Hospital, Singapore.
}

\section{Problem statement}

Parasitic fibroid is a rare phenomenon in which the fibroid gets partially or completely separated from the uterus and receives its blood supply from another source. This rare type of fibroid was first reported a century ago which was considered to have arisen due to torsion around its pedicle becoming separated from uterus and receiving blood supply from omentum or mesenteric vessels. But a sharp rise in the incidence of such cases in recent years following widespread use of laparoscopic power morcellation has thrown light on the iatrogenic etiology of parasitic fibroids.

\section{Methods}

Description of a case of multiple parasitic fibroids in different locations discovered in a woman few years after laparoscopic myomectomy.

\section{Results}

A 40 year old woman was seen as outpatient in Obstetrics and Gynaecology clinic, Singapore General hospital with complaints of palpable masses in the abdominal wall around the umbilical region. She had undergone laparoscopic myomectomy 7 years ago to remove a single $6 \mathrm{~cm}$ intramural fibroid from the anterior wall of the uterus. Ultrasound imaging revealed multiple discrete hypoechoic nodules ranging from $4.2 \mathrm{~cm}$ to $7.4 \mathrm{~cm}$ superior to the fundus and in the subcutaneous tissue in the umbilical region along with a $7.9 \mathrm{~cm}$ fibroid in the posterior wall of the uterus which suggested the diagnosis of multiple parasitic fibroids. She underwent laparotomy through a pfannensteil approach with removal of a large $8 \mathrm{~cm}$ pedunculated fibroid arising from the posterior peritoneal wall in the pouch of Douglas. Subsequently, through the same approach, three fibroids of about $7 \mathrm{~cm}, 4 \mathrm{~cm}$ and $3 \mathrm{~cm}$ were removed from the subrectus layer under the umbilicus followed by removal of a $2 \mathrm{~cm}$ fibroid from the subrectus layer in the suprapubic region. Her postoperative period was uneventful and got discharged from the hospital on the third postoperative day. The removed specimens were sent for histology and were confirmed to be fibroids.

\section{Conclusion}

Parasitic fibroid is a rare pathology often associated with laparoscopic morcellation but the possibility of having this uncommon complication should not deter surgeons from using morcellators in laparoscopic surgery. Careful patient selection, adequate patient counselling and surgeons adopting measures such as thorough inspection and repeated irrigation to remove all morcellated fragments during laparoscopy and preferably morcellation within an endoscopic bag can help to reduce the incidence of this iatrogenic complication.

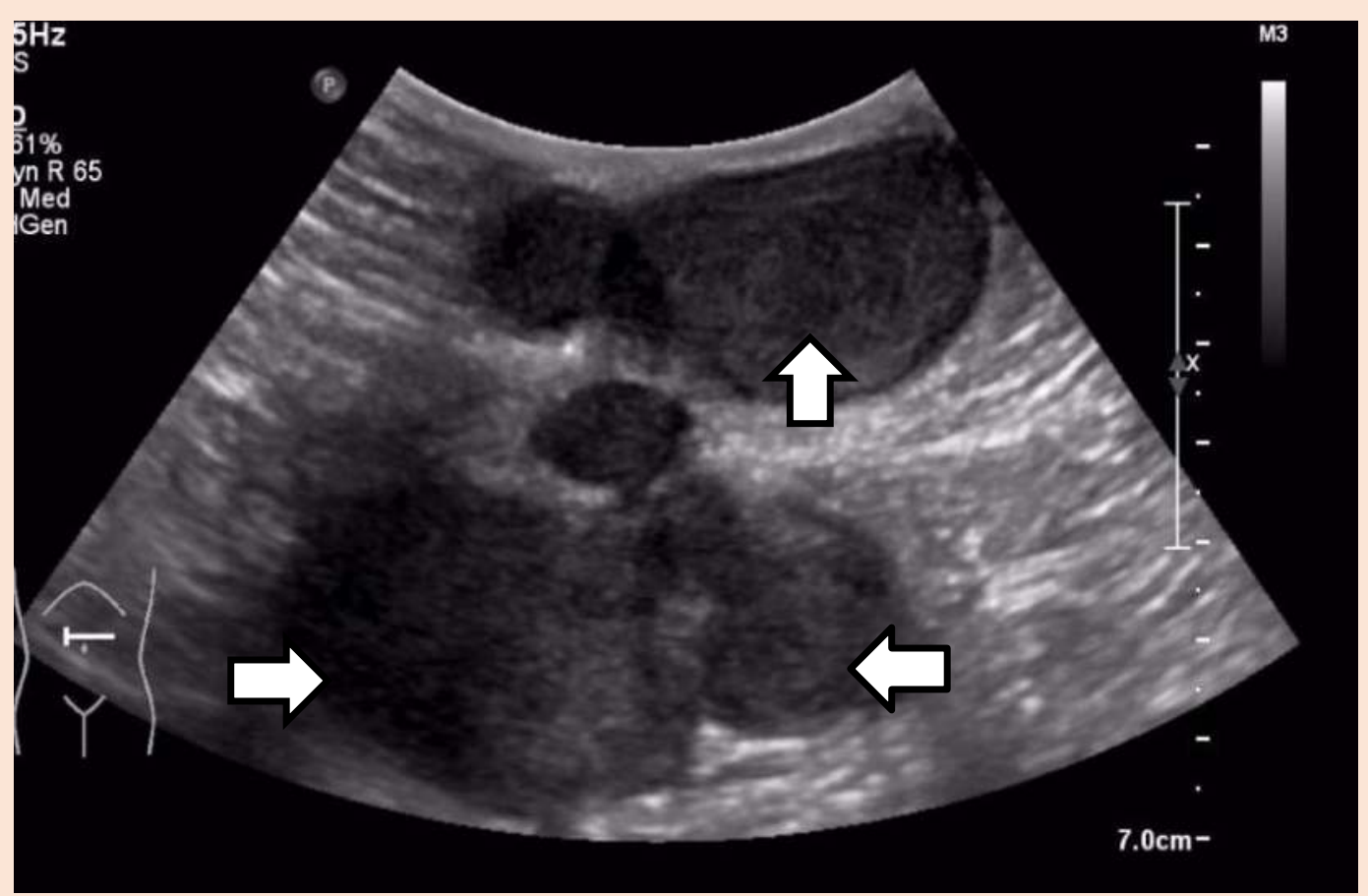

Abdominal wall masses on ultrasound

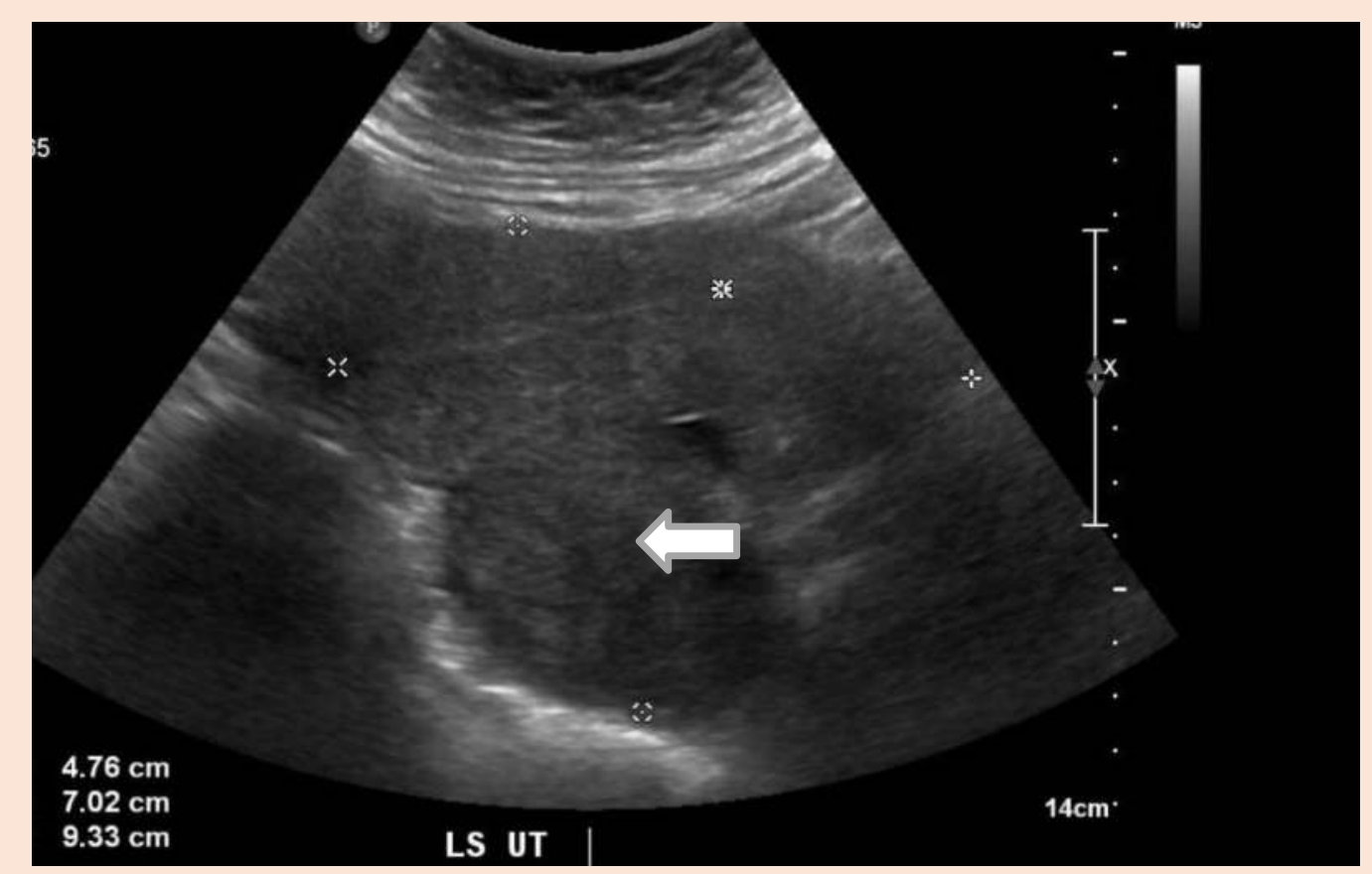

Parasitic fibroid in the pouch of Douglas

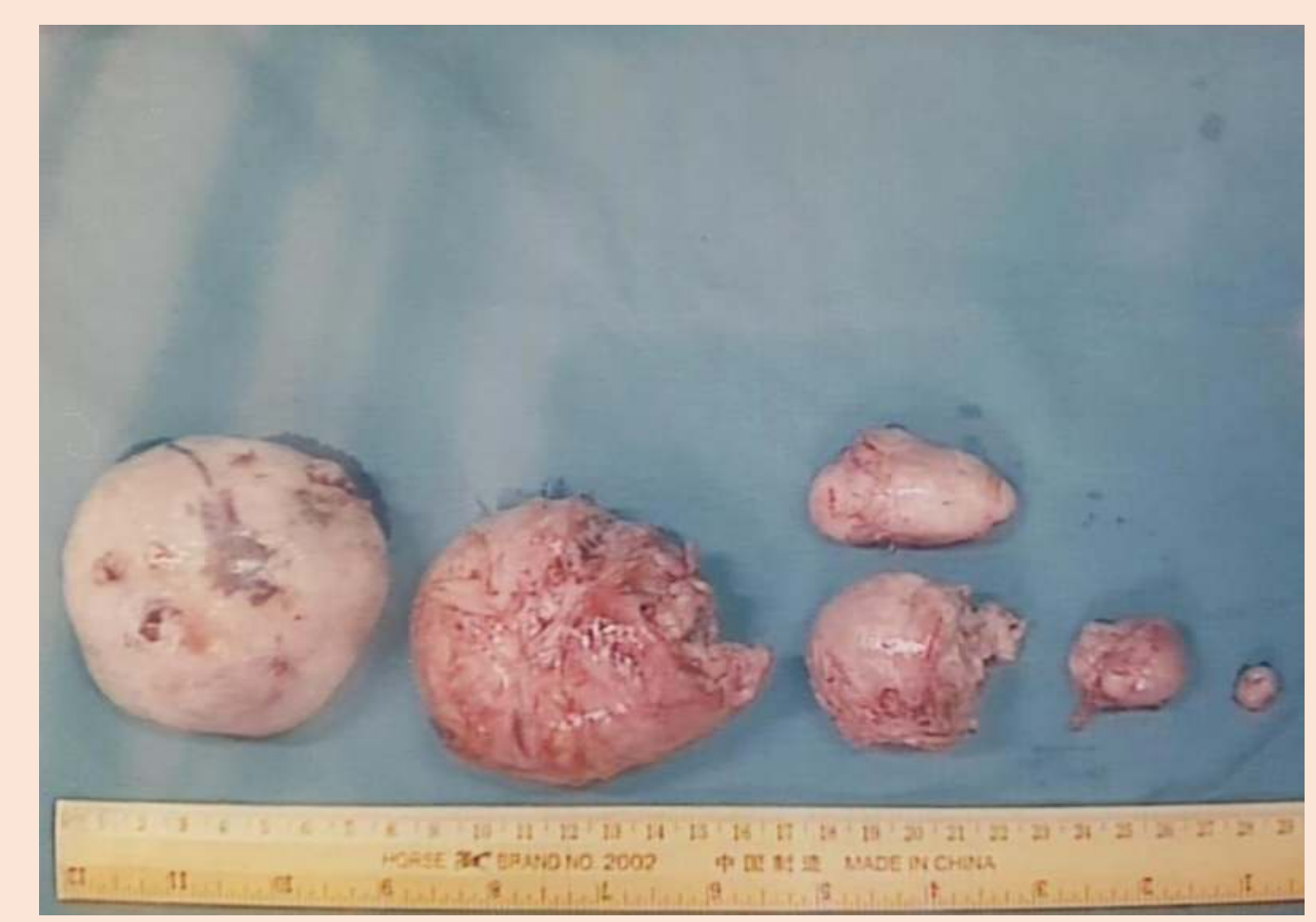

Parasitic fibroids removed during surgery 